\title{
Are tracheal surveillance cultures useful in the intensive care unit?
}

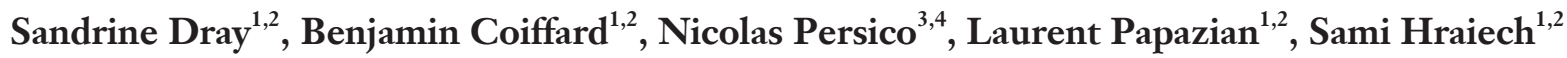 \\ ${ }^{1}$ Service de Médecine Intensive - Réanimation, APHM, Hôpital Nord, Marseille, France; ${ }^{2}$ CEReSS - Center for Studies and Research on Health \\ Services and Quality of Life EA3279, Aix-Marseille University, Marseille, France; ${ }^{3}$ Service d'Accueil des Urgences Adultes, ${ }^{4}$ Aix-Marseille Université, \\ Assistance Publique-Hôpitaux de Marseille, Hôpital Nord, Marseille, France \\ Contributions: (I) Conception and design: All authors; (II) Administrative support: S Hraiech, L Papazian; (III) Provision of study materials or patients: \\ All authors; (IV) Collection and assembly of data: All authors; (V) Data analysis and interpretation: S Dray, B Coiffard, N Persico, S Hraiech; (VI) \\ Manuscript writing: All authors; (VII) Final approval of manuscript: All authors. \\ Correspondence to: Sami Hraiech. Service de Médecine Intensive - Réanimation, APHM, Hôpital Nord, Marseille 13015, France. \\ Email: sami.hraiech@ap-hm.fr.
}

\begin{abstract}
Endotracheal aspirate (ETA) surveillance cultures have been used to predict the microorganisms responsible for ventilator associated pneumonia (VAP) in intensive care unit (ICU) patients for 3 decades. However, although more than a dozen studies have been performed, the usefulness and the safety of this strategy are still debated. Tracheobronchial bacterial colonization often precedes the occurrence of VAP, and it has been postulated that the microbes present in the tracheal secretions a few days before VAP might be the same as those retrieved in the lower respiratory tract. A large number of studies, with heterogeneous designs and variable results, have questioned the possibility of predicting, by regular ETA cultures after the $48^{\text {th }}$ hour of mechanical ventilation $(\mathrm{MV})$, the microbiology of VAP and therefore of determining the adequate antibiotic therapy to limit the over-prescription of broad spectrum molecules when following guidelines. Although it has shown some promising results, the strategy has not achieved unanimity because of some discordant data. The aim of this review is to provide an updated overview of the literature available in the field and to attempt to determine the strengths and weaknesses of antibiotic stewardship based on ETA surveillance cultures in VAP, particularly in the global context of drug resistant microorganism emergence and the crucial necessity of broad spectrum molecule preservation.
\end{abstract}

Keywords: Ventilator associated pneumonia (VAP); endotracheal aspirate (ETA); routine surveillance cultures; antibiotics; intensive care unit (ICU)

Submitted Jul 31, 2018. Accepted for publication Aug 17, 2018.

doi: $10.21037 /$ atm.2018.08.39

View this article at: http://dx.doi.org/10.21037/atm.2018.08.39

\section{Introduction and general context}

Pneumonia accounts for more than $60 \%$ of the infections diagnosed in intensive care unit (ICU) patients worldwide (1). Ventilator associated pneumonia (VAP) is at the forefront of nosocomial infections in the ICU and affects $10 \%$ to $20 \%$ of patients under mechanical ventilation (MV) for more than 48 hours (2). In Europe, more than half of the infections in ICU patients are VAP (3). In France, according to the report of the nosocomial infections surveillance network in intensive care (REA-Raisin 2012 report), the incidence of VAP between 2004 and 2012 oscillated around
$12.5 \%$ of patients under MV (density of incidence: 14.5 per 1,000 days of exposure).

The mortality due to VAP is assessed by varying means, mainly because of the difficulty of determining the proportion of deaths directly attributable to these infections (4-6) VAP-associated mortality ranges from $24 \%$ to $76 \%$ (4). However, the negative impact of VAP on the prognosis of ICU patients has been clearly demonstrated: VAP increases the duration of MV and the ICU stay by approximately 5 to 7 days and is a cause of increased consumption of antibiotics (7). VAP results in a significant additional cost of hospital care $(2,7,8)$. The occurrence of a VAP is associated 
with a higher consumption of hospital devices estimated between 5 and $40 \mathrm{~K} €$ per $\operatorname{VAP}(2,8,9)$.

The suspicion of VAP requires its confirmation and the identification of microorganisms to adapt the antibiotic treatment to the profile of susceptibility. For this, microbiological sampling with cultures of airway secretions is recommended before initiating antibiotic therapy $(10,11)$. Several methods of microbiological diagnosis have been evaluated, the most common being endotracheal aspirate (ETA), bronchoalveolar lavage (BAL), protected distal sampling (PDS) and telescopic brush. The positivity threshold of these samples varies according to their respective sensitivity and specificity. Nevertheless, current guidelines differ concerning the preferred diagnostic method. The American Thoracic Society (ATS)/ Infectious Diseases Society of America (IDSA) suggest the use of (10) so-called "non-invasive" samples (ETA) rather than invasive specimens (BAL, protected specimen brush), considering that the literature is not conclusive on the superiority of invasive techniques with respect to the patient's prognosis $(12,13)$. On the other hand, the European Respiratory Society (ERS)/European Society of Intensive Care Medicine (ESICM)/European Society of Clinical Microbiology and Infectious Diseases (ESCMID)/ Latin American Thoracic Association (ALAT) (11) suggest obtaining distal quantitative samples to reduce antibiotic exposure in stable patients with suspected VAP and to improve the accuracy of the results. Both of these guidelines are weak recommendations, with low quality of evidence.

Regardless of the diagnostic method, the delay necessary to obtain the sample's culture requires the initiation of broad spectrum probabilistic antibiotic therapy to treat all the bacteria that are potentially involved $(10,14)$, leading to the prescription of molecules that favour the emergence of multi-drug resistant (MDR) bacteria, such as 3 rd or 4th generation cephalosporins, carbapenems, fluoroquinolones or drugs (oxazolidinones, vancomycin) with activity against methicillin resistant Staphylococcus aureus (MRSA) (10,14). Some of these drugs also represent a significant cost. Moreover, in a non-negligible proportion of cases, these broad spectrum antibiotics are prescribed unnecessarily, with VAP being ultimately not confirmed. The over-prescription of antibiotics is one of the main factors responsible for the emergence of MDR strains, and this increase in the number of resistant organisms is itself the cause of an escalation in the prescription of broadspectrum antibiotics (15). The emergence of MDR strains, and especially carbapenem-resistant Enterobacteriaceae (16) or beta-lactam-resistant Pseudomonas aeruginosa, represents a global threat, especially considering the scarcity of new molecules in the pipeline. In this context, reducing the length of antibiotic treatments and de-escalation have been recommended in the care of $\operatorname{VAP}(10,11)$. Recently published data showed that a reduction in the length of antibiotic treatments (guided by procalcitonin kinetics) was associated with a decrease in short- and long-term mortality (17). Additionally, other studies evaluating the cost and efficacy of procalcitonin to guide antibiotic therapy have shown that a reduced duration of antibiotic treatment led to reduced hospital costs $(18,19)$. It is therefore crucial, for medical reasons, to reduce antibiotic use and, from an economic point of view, to have a rigorous stewardship of antibiotics for VAP.

In this context, one alternative to broad spectrum probabilistic antibiotics is the adaptation of VAP treatment to the microbial flora colonizing the patients' upper respiratory tract. This method, based on regular surveillance of ETA, was first described in the early 1990's (20) and has been investigated in several studies since then. The dramatic context of bacterial resistance emergence could renew interest in this technique.

\section{From tracheal colonization (TC) to VAP: physiopathological aspects}

The literature largely suggests an association between the occurrence of TC in the ICU and the subsequent development of VAP with the same microorganisms $(21,22)$. In healthy subjects, the airway is sterile and protects itself from constant exposure to microorganisms to protect the respiratory tract from colonization or invasion by environmental microbes. Several mechanisms are involved, such as anatomic barriers (the glottis and larynx), cough reflex, mucociliary clearance, phagocytosis by resident macrophages, and respiratory secretions. These mechanisms have strong antimicrobial capacities due to multiple factors including lysozyme, lactoferrin, and secretory leukoproteinase inhibitor (23).

When the barriers and mechanisms of host defence are affected, potentially pathogenic microorganisms might occur in the lower respiratory tract. This phenomenon is called tracheal or tracheobronchial colonization and is defined as the presence of microorganisms in cultures of the trachea samples in the absence of clinical signs of respiratory infection. TC is common among critically ill patients requiring intubation and MV. The incidence of TC 
varies widely in the literature from $20 \%$ to almost $100 \%$ of mechanically ventilated patients (24-26). This colonization generally occurs early after intubation, often within the first 24 hours (27).

Several routes of TC have been proposed: primary TC without previous colonization in other sites (28), aspiration of colonized secretions from the oropharynx (29), and/ or aspiration of colonized gastric contents (30). For the latter, in this hypothesis, microorganisms initially colonize the stomach, subsequently develop in the upper respiratory tract, and finally are aspirated into the lungs. Gastric colonization might be stimulated by decreased gastric acidity, as a result of stress ulcer prophylaxis or gastric nutrition. Many other factors were demonstrated to influence TC and VAP incidences, including antimicrobial treatment, the duration of $\mathrm{MV}$, reintubation, methods used to collect respiratory secretions, frequency of respiratory specimen collection (i.e., daily $v s$. twice or thrice weekly), sedation, paralytic agents, supine position and host factors [elderly, chronic obstructive pulmonary disease (COPD), coma, trauma, burns, organ failure, sinusitis...] $(4,22,24)$. The endotracheal tube can also become colonized, a process likely facilitated by biofilm formation (31). Biofilm accumulates on the tube, providing a protected environment for pathogens, and can become detached spontaneously or during suctioning or bronchoscopy.

The aetiology and microorganisms responsible for TC and VAP depend on multiple factors mentioned above such as the duration of $\mathrm{MV}$, prior administration of antibiotics, presence of COPD, and local factors. Gramnegative bacteria (GNB) and Staphylococcus aureus are the most common agents but the proportion might widely differ according to the characteristics of the geographic area and ICU. GNB, such as Pseudomonas aeruginosa, Acinetobacter spp. and Enterobacteriaceae, represent $55 \%$ to $85 \%$ of VAP cases. Staphylococcus aureus is involved in $20 \%$ to $30 \%$ of incidences of trachea-bronchial colonization and infection. Forty percent to $60 \%$ of VAP are polymicrobial infections (32).

Despite the somewhat different definitions of early-onset pneumonia, high rates of Haemophilus influenzae, Streptococcus pneumoniae, methicillin susceptible Staphylococcus aureus (MSSA), or susceptible Enterobacteriaceae were constantly found in early-onset VAP, whereas Pseudomonas aeruginosa, Acinetobacter spp., methicillin resistant Staphylococcus aureus (MRSA), and MDR GNB were significantly more frequent in late-onset VAP $(32,33)$. It is now well known that during critical illness, especially in ICU patients, the oral flora shifts dramatically to a predominance of aerobic Gram-negative bacilli and Staphylococcus aureus. Ewig et al. found that, upon admission to the ICU, patients were colonized mainly with S. aureus, Haemophilus influenzae, and Streptococcus pneumoniae; however, followup cultures showed rapid replacement of the normal oropharyngeal flora with enteric Gram-negative bacilli and $P$. aeruginosa (22). This different distribution pattern of aetiological agents between early- and late-onset VAP is also linked to the frequent administration of prior antimicrobial therapy in many patients with late-onset VAP.

Despite strong strategies to prevent VAP, tracheal colonization might occur and favour nosocomial pneumonia. As suggested by the infrequent association of VAP with bacteraemia, the majority of these infections appear to result from the aspiration of potential pathogens that have colonized the mucosal surfaces of the oropharyngeal airways (34). Pneumonia results from the microbial invasion of the normally sterile lower respiratory tract and lung parenchyma caused by a defect in host defences, challenge by a particularly virulent microorganism, or a substantial inoculum. Experimental investigations have linked some of the risk factors of VAP listed above to changes in the adherence of GNB to respiratory epithelial cells. Although formerly attributed to losses of cell surface fibronectin, these changes in adherence more likely reflect alterations of cell surface carbohydrates, which might facilitate bacterial adhesion.

The aspiration of contaminated oropharyngeal, gastric, or tracheal secretions around the cuffed endotracheal tube into the normally sterile lower respiratory tract results in most cases of TC. Over time, bacterial concentrations and inflammation increase in mechanically ventilated patients, resulting in a greater risk of progression to VAP. The complex interactions between the patient's host defences $v s$. the quantity and virulence of the bacterial pathogen(s) entering the lower respiratory tract determines if colonization progresses to VAP (35).

There is a probable continuum process that ranges from airway colonization to ventilator associated tracheobronchitis (VAT) and VAP (36). Even if VAP occurs without previous airway colonization or VAT, colonization of the upper respiratory tract is one of the most important risk factors for VAP, especially VAP caused by Enterobacteriaceae or Pseudomonas species (24). Oropharyngeal colonization has been shown to be a powerful independent predictor of subsequent tracheobronchial colonization and a large portion of the pathogens isolated from patients with 
VAP were previously recovered from the oropharynx (37).

The first study describing regular surveillance of bronchial microflora in patients requiring MV using nondirected bronchial lavage quantitative cultures showed that during the days immediately before VAP, a significant increase in bacterial counts was detected (20). Taken together, all these elements led to the idea that routine surveillance of tracheal aspirates could be of interest to facilitate microbiological diagnosis and guide the treatment of VAP and consequently led to several trials to investigate this hypothesis.

\section{Usefulness of routine ETA for VAP diagnosis: performance and limits}

We tried to identify the strengths and weaknesses of ETA cultures surveillance-based antibiotic therapy for VAP according to the literature data. We focused on adult studies designed to investigate the following:

(I) correlation between the bacteria identified during the routine monitoring of ETA and those responsible for VAP;

(II) antimicrobial treatment adequacy.

The results are presented according to 6 clinically relevant endpoints.

\section{Microbiological diagnosis}

In a prospective observational study performed in a French medical ICU, Michel et al. (38) evaluated the concordance between the results of ETA routinely performed and those of the BAL carried out at the time of the suspicion of VAP. The authors conducted a biweekly monitoring of ETA cultures in 299 patients under MV for more than 48 hours. The diagnosis of VAP was made by BAL culture, and only the ETA performed just before the suspicion of VAP was taken into consideration. Sensitivities were determined for microorganisms present at a concentration of $\geq 10^{3}$ for ETA and $\geq 10^{4} \mathrm{CFU} / \mathrm{mL}$ for BAL. The authors compared the antibiotics prescribed following the ETA results to those that would have been prescribed if the ATS recommendations had been applied. VAP was diagnosed in 41 of the 75 patients with VAP suspicion. Among the 41 BAL specimens that were positive, ETA and BAL had identified the same microorganisms (with the same antibiotic resistance patterns) in 34 cases (83\%; 95\% CI: 70-96\%) considering only the last ETA performed before VAP. This concordance was even higher when considering only late-onset VAP. The last ETA before VAP was positive in 39/41 (95\%) patients in whom VAP was confirmed. Antibiotic therapy was delayed in 2 patients in which ETA ignored a VAP that was confirmed on BAL. ETA preceding VAP suspicion was sterile in $22 / 34(65 \%)$ patients in whom BAL finally confirmed the VAP suspicion.

In a prospective analysis of retrospective data, Jung et al. (39) evaluated the impact of a routine weekly EA in medical and surgical patients who had VAP confirmed by BAL. Of 113 VAP cases analysed, 90 ETA-BAL samples were assessable. Only the last ETA collected before the BAL was considered. The first finding of the study was that the most recent ETA before clinical suspicion of VAP and BAL were concordant (the same microorganisms with the same antibiotic resistance pattern) in $72 \%$ of patients $(65 / 90)$. There was no difference between early and lateonset pneumonia [concordance rate 25/33 (75\%) vs. $40 / 50$ $(80 \%)$, respectively]. Of note, antibiotics at the time of ETA did not impact the concordance with BAL. In the $28 \%$ patients with ETA/BAL discordance, the main reason was the absence of growth in ETA culture (not enough time between last ETA and VAP occurrence). Finally, the time between the last ETA and the BAL did not affect the rate of concordance $(\mathrm{P}>0.99)$. These data were not supported in most other studies.

Different results were found in a prospective, observational, cohort study by Luna et al. (40) conducted in a 15-bed ICU in Argentina and including 283 patients mechanically ventilated for $\geq 48 \mathrm{~h}$. The authors compared a strategy based on routine ETA to probabilistic antibiotics based on the ATS/IDSA in VAP. Interventions included twice-weekly ETA, and BAL was performed if VAP was suspected. The results of the ETA cultures were not routinely given to the attending physicians, as it was not part of the standard of care. One hundred and fortysix BAL (collected at the time of VAP) plus ETA culture (collected $\leq 7$ days before VAP) pairs were defined. The authors compared two models of 10 days of empirical antimicrobials (ETA-based vs. ATS/IDSA guidelines-based strategies), analysing their impact on the appropriateness of therapy and total antimicrobial-days, using the BAL result as the standard for comparison. Overall, ETA predicted the pathogens in $62.4 \%$ of all pairs. The concordance was only $46.2 \%$ of pairs if ETA was performed 3 to 7 days before BAL but increased to $74.0 \%$ of pairs if ETA was performed $<3$ days before BAL $(\mathrm{P}=0.016)$. The ETA/BAL concordance increased to $86.7 \%$ for the diagnosis of a subsequent episode of BAL and when the last ETA available had been 
performed less than 3 days before VAP.

In 53 burn patients with inhalation injury necessitating MV, Brusselaers et al. (41) evaluated the role of routine surveillance of ETA performed thrice weekly. Microbiological confirmation of VAP required semiquantitative growth of a pathogen. The authors found the sensitivity and specificity of the surveillance cultures to be $83.3 \%$ (95\% CI: $64.1-93.3$ ) and $96.2 \%$ (95\% CI: 89.4-98.7), respectively. When calculated based on the total number of VAP episodes $(\mathrm{n}=70)$ instead of the total number of microorganisms $(\mathrm{n}=101)$, the sensitivity and specificity were slightly lower (respectively $82.6 \%$ and $95.7 \%$ ) and the positive and negative predictive values (NPV) were higher (respectively $90.5 \%$ and $91.8 \%$ ).

Brusselaers et al. (42) performed a systematic review and a diagnostic test accuracy meta-analysis in which they analysed the sensitivity and specificity of surveillance cultures in predicting pathogens in VAP. Fourteen studies were included, showing a high accuracy of surveillance cultures, with pooled sensitivities up to 0.75 and specificities up to 0.92 in culture-positive VAP. They recovered a sensitivity of $78 \%$ and a specificity of $96 \%$ of an ETA monitoring performed twice weekly when focusing solely on the most recent ETA before VAP. No additional benefit could be demonstrated for thrice weekly sampling.

These last results argue for a good diagnostic performance of routine ETA surveillance cultures under specific conditions (be-weekly sample performance, considering only the last ETA results). However, clinical and statistical heterogeneity, limited sample sizes, and bias remain important limitations of this meta-analysis.

\section{Drug resistance diagnosis}

In a retrospective observational study investigating the usefulness of periodical ETA to identify the bacteria responsible for subsequent late onset of VAP (35), the positive predictive values for $P$. aeruginosa and MRSA from routine ETA samples were $92 \%$ and $90 \%$, respectively. The NPV for $P$. aeruginosa and MRSA from routine ETA samples were $75 \%$ and $89 \%$, respectively. Chan et al. (43) also found a high NPV of ETA routine surveillance for the diagnosis of MRSA in VAP.

In Brusselaers et al.'s study (41), 23 MDR pathogens were isolated in surveillance culture, and 20 of these were found in the VAP as well. Routine ETA surveillance cultures predicted MDR pathogens with an $87 \%$ PPV and a $95 \% \mathrm{NPV}$, in particular for $P$. aeruginosa and MDR

\section{Enterobacteriaceae.}

Finally, in the meta-analysis by Brusselaers et al. (42), the presence of MDR microorganisms in surveillance cultures of the lower respiratory tract predicted the pathogen(s) with a $75-82 \%$ confidence rate. Overall, ETA surveillance appears to be a reliable tool to diagnose and to exclude the implication of MDR pathogens in VAP.

\section{Antimicrobial treatment adequacy}

In a Michel et al. study (38), routine ETA performed twice a week made it possible to prescribe adequate antibiotic therapy in $95 \%$ of the patients in whom a VAP was ultimately diagnosed by BAL. ETA-based treatment was superior to a probabilistic strategy based on the ATS guidelines $(95 \%$ vs. $68 \%, \mathrm{P}=0.005)$. With only one ETA per week, Jung et al. (39) found that $85 \%$ of the prescribed probabilistic antibiotic therapies were adequate, compared to $73 \%(\mathrm{P}<0.05)$ for antibiotic therapies based on the most recent ATS recommendations. In this study, ATS strategy failure was mainly due to beta lactam $P$. aeruginosa resistance, MRSA and extended spectrum beta-lactamases (ESBL) Enterobacteriaceae. However, Luna et al. (40) found that a strategy based on the ATS guidelines was more efficient in guiding antibiotic treatment (appropriate therapy in $97.9 \%$ and $82.2 \%$ of pairs, respectively, for ATS strategy and ETA surveillance; $\mathrm{P}<0.002$ ), with the difference between the 2 strategies being smaller when the time between the last ETA available and the BAL was less than 3 days. In this study, inappropriate therapy would have been no therapy for the first 2 days before BAL data were available (false negative) or wrong or incomplete antimicrobial coverage.

\section{Antibiotics sparing}

In Michel et al.'s (38) study, when the last ETA preceding the suspicion of VAP was sterile, it was possible to avoid starting a probabilistic antibiotic therapy in $95 \%$ of cases, the BAL finally returning sterile too. Based on ETA results, the use of carbapenems, anti-pseudomonal B-lactams was restricted to $45 \%$ patients, whereas they would have been used in $80 \%$ patients if the ATS recommendations had been used $(\mathrm{P}<0.002)$. Antibiotics were prescribed (based on the ETA-pre VAP results) in excess in 8 patients in whom VAP was not confirmed by BAL. In these "false positive" patients, based on the ATS-based strategy, a broad spectrum antibiotic would have been used in $91 \%$ of patients. In 
a Luna et al. (40) study, the ETA-based strategy would have led to the prescription of $24.4 \%$ fewer antimicrobialdays than the ATS/IDSA guidelines-based strategy. Deescalation was possible in $95 \%$ of patients following the ATS strategy, compared to only $40 \%$ of patients following ETA surveillance.

\section{Clinical outcomes}

No study has compared the outcomes of patients with VAP being treated according to ETA surveillance or guidelines. Jung et al. (39) found that the concordance or discordance between ETA and BAL did not affect ICU mortality. The study was not designed to demonstrate an effect on mortality or other clinical endpoints with the strategy of treatment guided by pre-VAP ETA.

\section{Cost effectiveness ratio}

The economic aspect of an ETA surveillance strategy was briefly reported by Michel et al. (38). The total cost of the antibiotics administered while waiting for BAL culture results (based on the ETA-pre VAP culture results) and a routine ETA specimen culture performed twice a week for all patients who received MV for more than 48 hours during the study period was significantly higher than empirical treatment with imipenem, amikacin, and vancomycin for 48 hours (while waiting for the BAL culture result) for all patients who were suspected of having VAP. However, these results did not take into account the potential impact of the ETA surveillance strategy on shortening treatments and decreasing overall antibiotic consumption.

\section{Synthesis and perspectives}

\section{Key messages from the literature}

Even if randomized control trials (RCTs) are lacking to strengthen the evidence, some key messages can be drawn from the literature.

First, routine ETA surveillance appears to be able to successfully predict the microorganisms responsible for VAP in a large portion of cases. However, the performance of such a strategy is influenced by several factors that are linked to the patient and the technique, which probably explains the discrepancies in the results of some studies. Twice weekly ETA is likely necessary to limit the time between the availability of the last sample and the day of
VAP occurrence. Indeed, the diagnostic performance of the strategy clearly decreases when this duration is 3 days or more. Moreover, the method performs better when considering only the most recent culture and subsequent episodes of VAP rather than in early-onset VAP, when TC colonization is not yet present. Under these conditions, routine surveillance of ETA appears to be a reliable tool, especially to exclude an uncertain diagnosis of VAP or to predict a VAP involving MDR strains, including MRSA. The high sensitivity and specificity of the technique could be used to question the diagnosis of VAP when the last ETA performed in the abovementioned conditions is sterile. This could lead to two major consequences as follows: the search for another site of infection with the realization of large microbiological sampling and/or the absence of prescription of antibiotic treatments. Of course, the safety of such an approach needs to be first investigated in a RCT. One of the main limitations of the design of such a study would be the exclusion of patients with septic shock or worsening ARDS in whom delayed antibiotic treatment would be unacceptable.

Second, as a consequence of the diagnostic performance of the technique, it appears reasonably feasible to guide the probabilistic antibiotic treatment according to the susceptibility profile of the bacteria retrieved in the last ETA. This is of crucial importance especially for ESBL Enterobacteriaceae or non-fermenting GNB such as $P$. aeruginosa or Acinetobacter species, which are frequently associated with inadequate antibiotic treatments and increased mortality $(44,45)$. Routine surveillance antibiotic adaptation demonstrated a higher adequacy than guideline based treatment concerning these microorganisms in several studies (38,39).

Third, ETA surveillance could allow for limiting the prescription of broad spectrum molecules, in particular sparing carbapenems, which is of crucial interest in this period of drug scarcity and carbapenem-resistant bacteria emergence. Of note, the literature is consistent in this field. Even the work from Luna et al. (40), which is conclusive on the superiority of guidelines for the strategy concerning antibiotics adequacy, highlights the fact that de-escalation was possible in $95 \%$ of cases when using the ATS guidelines strategy but in only $40 \%$ of cases when following ETA surveillance, allowing 3 days or more of broad spectrum antibiotic saving. The same study found a significant reduction in the antimicrobial days in the ETA-based strategy.

What is less clear is the cost-effectiveness ratio of the 
strategy. The economic evaluation of routine diagnostic tests followed by early targeted antibiotic therapies has not been evaluated. It would be necessary to evaluate the balance between the possible savings achieved as a result of the reduction in antibiotic use and the additional cost of prescribing ETA twice a week in all patients with MV for more than 48 hours. A medico-economic study with a methodology specifically designed to evaluate both health benefits and costs may provide the necessary information on the cost efficiency of such a strategy. It is possible that the additional costs of sampling and diagnosis would be counterbalanced by the avoided costs related to decreased antibiotic consumption, in particular of expensive drugs, and perhaps a decrease in the length of the ICU stay.

\section{Potential benefits of the ETA strategy}

Systematic monitoring of ETA could reduce the number of days with antibiotics and limit the use of broad-spectrum molecules. Direct benefits could be expected for the patient, mainly the reduction of the risk of colonization and infections due to MDR bacteria. Antibiotic stewardship has also been shown to improve the outcomes of patients with Clostridium difficile infections (46). Moreover, some studies have demonstrated that de-escalation was associated with a decrease in mortality (47-49) and ICU length of stay (49), even if conflicting data exist about these endpoints.

A strategy based on ETA monitoring could be generalized and help improve the quality and safety of health care systems and decrease health costs.

\section{Limitations}

Two factors must be considered and probably explain why current guidelines do not include ETA surveillance as a potential strategy to limit antibiotic consumption in the ICU.

The first limitation is the conflicting data concerning the adequacy of microbiological diagnosis and, consequently, the accuracy of antibiotic treatment. The main concern raised by some authors is the risk of VAP diagnosis misleading and causing under-treatment in the ETA strategy. However, the meta-analysis from Brusselaers et al. (42) concluded with coherent evidence for the accuracy of surveillance cultures of the respiratory tract to predict bacterial pathogens in VAP, in particular, the absence of multidrug-resistant pathogens. Moreover, the most important clinical implication is the high specificity and negative likelihood of surveillance cultures. In other words, if a recent surveillance culture does not contain MDR microorganisms, the newly symptomatic VAP is unlikely to be caused by MDR. This message must be tempered considering the clinical and statistical heterogeneity and limited samples sizes of the studies included in the metaanalysis.

The second limitation, and the main barrier, is the absence of a RCT evaluating the impact of the ETA strategy on the prognosis of ICU patients. To date, all of the studies have focused on the microbiological accuracy and the ability of antibiotic sparing. However, the prerequisite for the large diffusion of the ETA strategy is the demonstration of its safety compared to the guidelines strategy. Before being largely advised, ETA surveillance cultures must demonstrate, using a strong design, that an increase in mortality or morbidity is not the price to pay for potential beneficial effects of antibiotic use.

\section{Perspectives}

No study has primarily evaluated the clinical impact of such a strategy on the prognosis of ICU patients. It could be of interest to evaluate if systematic and bi-weekly ETA monitoring could reduce the global use of antibiotics and limit the prescription of broad spectrum molecules during VAP suspicion without increasing mortality compared to a probabilistic strategy of antibiotic prescriptions based on guidelines. Such a study would also assess the efficacy of ETA routine surveillance on the duration of $M V$ and ICU as well as hospital stay, antibiotic consumption, broad-spectrum antibiotic use, incidence of infections or colonization involving MDR strains, and medico-economic analysis impact of the strategy.

Despite the lack of data from a RCT, routine surveillance of ETA might be one way to limit over-prescription of broad spectrum antibiotics. In Figure 1, we provide an illustration of a potential prescription algorithm based on ETA surveillance cultures. Immunosuppressed patients (severely neutropenic, AIDS patients) would not be affected by this strategy. Moreover, because of the (minor) risk of antibiotic delay in cases of sterile last ETA but positive BAL, septic shock or worsening ARDS patients should not be managed following the algorithm. A meaningful number of patients with suspicion of VAP and without criteria of severity could however be concerned by such a protocol. 
A
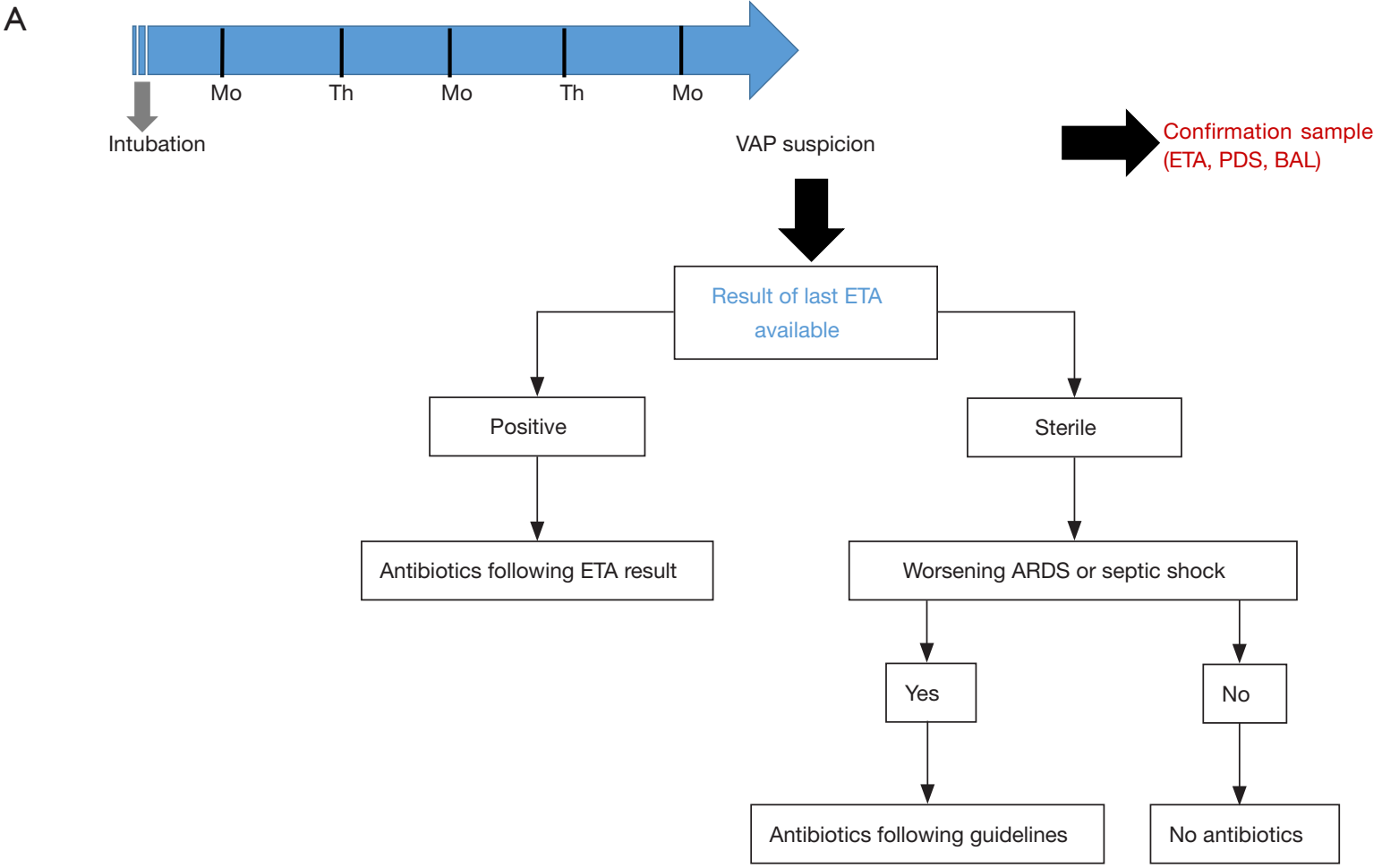

B

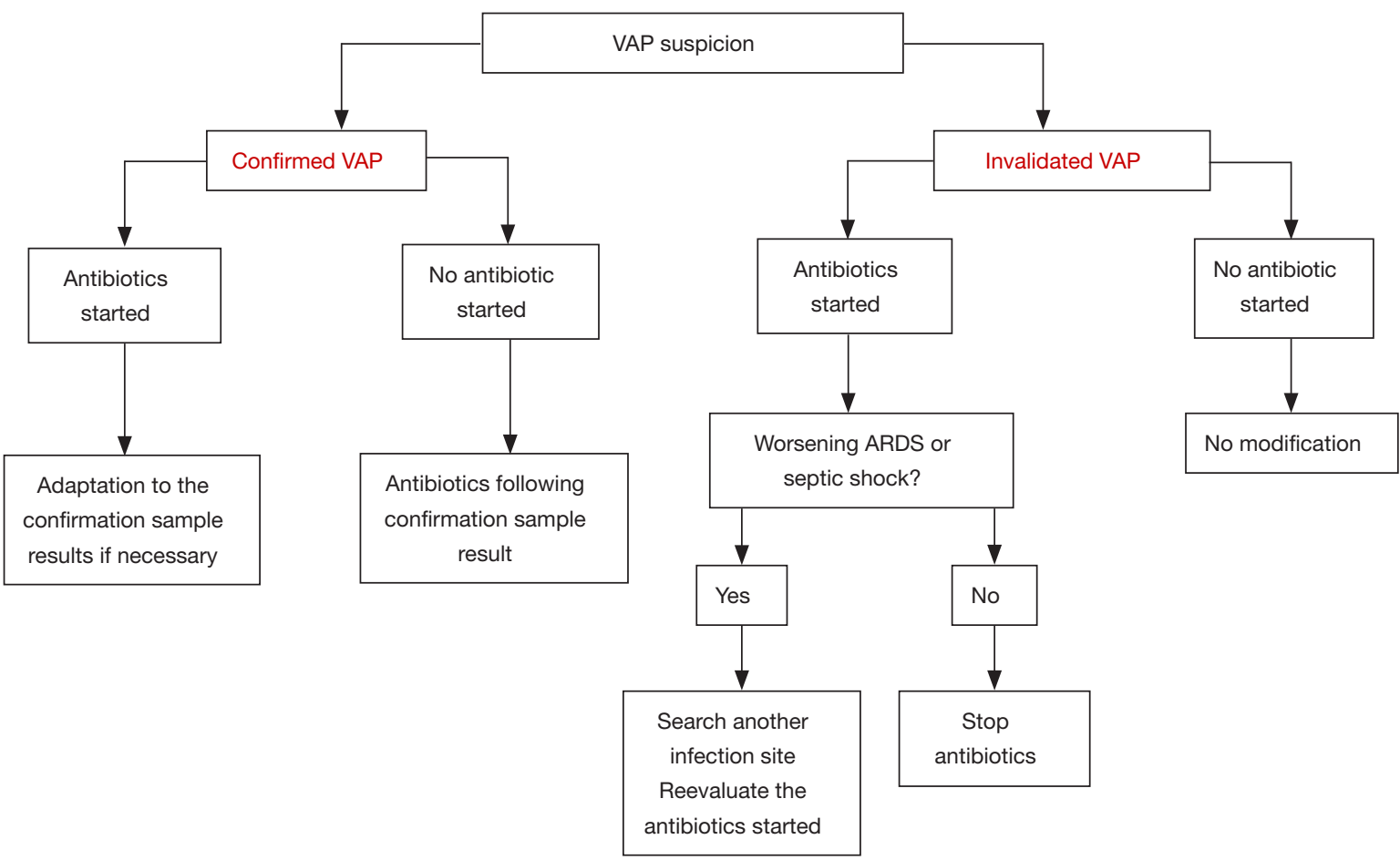

Figure 1 A proposed algorithm of VAP antibiotic prescription based on ETA surveillance cultures. (A) In this example, ETAs are routinely performed on Monday and Thursday after the $48^{\text {th }}$ hour of MV. In cases of VAP suspicion, a confirmation sample is performed to confirm or not confirm the VAP-onset. Antibiotics are prescribed according to the results of the most recent ETA available. Immunocompromised patients should not be affected by this algorithm; (B) propositional strategy after VAP confirmation or non-confirmation. Mo, Monday; Th, Thursday; ETA, endotracheal aspirate; PDS, protected distal sample; BAL, broncho-alveolar lavage; VAP, ventilator-associated pneumonia; ARDS, acute respiratory distress syndrome. 
Finally, the main practical limit to the execution of such a strategy could be the time to obtain the culture results of ETA. In real life, it is often difficult to obtain these results within 2 or 3 days, and the technique performance clearly decreases when relying on older sampling. The recent advances in bacterial identification, such as molecular biology and/or mass-spectrometry, might eliminate this weakness and renew interest in the routine surveillance of ETA.

\section{Conclusions}

Several studies have demonstrated that, providing some conditions, ETA can be routinely performed to reliably predict the microorganisms of VAP. However, the literature is inconsistent, and a confirmation study with a robust methodology and focusing on the patient's prognosis is still lacking to definitively conclude and recommend the widespread use of routine surveillance of tracheobronchial flora. The growing challenge of broad spectrum antibiotics sparing combined with the development of microbial rapid identification tools might open the way to the revival of this strategy.

\section{Acknowledgements}

None.

\section{Footnote}

Conflicts of Interest: The authors have no conflicts of interest to declare.

\section{References}

1. Vincent JL, Rello J, Marshall J, et al. International study of the prevalence and outcomes of infection in intensive care units. JAMA 2009;302:2323-9.

2. Safdar N, Dezfulian C, Collard HR, et al. Clinical and economic consequences of ventilator-associated pneumonia: a systematic review. Crit Care Med 2005;33:2184-93.

3. Vincent JL, Bihari DJ, Suter PM, et al. The prevalence of nosocomial infection in intensive care units in Europe. Results of the European Prevalence of Infection in Intensive Care (EPIC) Study. JAMA 1995;274:639-44.

4. Chastre J, Fagon JY. Ventilator-associated pneumonia. Am J Respir Crit Care Med 2002;165:867-903.
5. Timsit JF, Chevret S, Valcke J, et al. Mortality of nosocomial pneumonia in ventilated patients: influence of diagnostic tools. Am J Respir Crit Care Med 1996;154:116-23.

6. Melsen WG, Rovers MM, Groenwold RH, et al. Attributable mortality of ventilator-associated pneumonia: a meta-analysis of individual patient data from randomised prevention studies. Lancet Infect Dis 2013;13:665-71.

7. Rello J, Ollendorf DA, Oster G, et al. Epidemiology and outcomes of ventilator-associated pneumonia in a large US database. Chest 2002;122:2115-21.

8. Warren DK, Shukla SJ, Olsen MA, et al. Outcome and attributable cost of ventilator-associated pneumonia among intensive care unit patients in a suburban medical center. Crit Care Med 2003;31:1312-7.

9. Kollef MH, Hamilton CW, Ernst FR. Economic impact of ventilator-associated pneumonia in a large matched cohort. Infect Control Hosp Epidemiol 2012;33:250-6.

10. Kalil AC, Metersky ML, Klompas M, et al. Management of Adults With Hospital-acquired and Ventilator-associated Pneumonia: 2016 Clinical Practice Guidelines by the Infectious Diseases Society of America and the American Thoracic Society. Clin Infect Dis 2016;63:61-111.

11. Torres A, Niederman M, Chastre J, et al. International ERS/ESICM/ESCMID/ALAT guidelines for the management of hospital-acquired pneumonia and ventilator-associated pneumonia. Eur Respir J 2017;50:1700582.

12. Fagon JY, Chastre J, Wolff $M$, et al. Invasive and noninvasive strategies for management of suspected ventilator-associated pneumonia. A randomized trial. Ann Intern Med 2000;132:621-30.

13. Canadian Critical Care Trials Group. A randomized trial of diagnostic techniques for ventilator-associated pneumonia. N Engl J Med 2006;355:2619-30.

14. American Thoracic Society, Infectious Diseases Society of America. Guidelines for the management of adults with hospital-acquired, ventilator-associated, and healthcareassociated pneumonia. Am J Respir Crit Care Med 2005;171:388-416.

15. Meyer E, Schwab F, Schroeren-Boersch B, et al. Dramatic increase of third-generation cephalosporin-resistant E. coli in German intensive care units: secular trends in antibiotic drug use and bacterial resistance, 2001 to 2008. Crit Care 2010;14:R113.

16. Logan LK, Weinstein RA. The Epidemiology of Carbapenem-Resistant Enterobacteriaceae: The Impact and Evolution of a Global Menace. J Infect Dis 
2017;215:S28-36.

17. de Jong E, van Oers JA, Beishuizen A, et al. Efficacy and safety of procalcitonin guidance in reducing the duration of antibiotic treatment in critically ill patients: a randomised, controlled, open-label trial. Lancet Infect Dis 2016;16:819-27.

18. Christ-Crain M, Stolz D, Bingisser R, et al. Procalcitonin guidance of antibiotic therapy in community-acquired pneumonia: a randomized trial. Am J Respir Crit Care Med 2006;174:84-93.

19. Christ-Crain M, Jaccard-Stolz D, Bingisser R, et al. Effect of procalcitonin-guided treatment on antibiotic use and outcome in lower respiratory tract infections: clusterrandomised, single-blinded intervention trial. Lancet 2004;363:600-7.

20. A'Court CH, Garrard CS, Crook D, et al. Microbiological lung surveillance in mechanically ventilated patients, using non-directed bronchial lavage and quantitative culture. Q J Med 1993;86:635-48.

21. Cardeñosa Cendrero JA, Solé-Violán J, Bordes Benítez A, et al. Role of different routes of tracheal colonization in the development of pneumonia in patients receiving mechanical ventilation. Chest 1999;116:462-70.

22. Ewig S, Torres A, El-Ebiary M, et al. Bacterial colonization patterns in mechanically ventilated patients with traumatic and medical head injury. Incidence, risk factors, and association with ventilator-associated pneumonia. Am J Respir Crit Care Med 1999;159:188-98.

23. Revenis ME, Kaliner MA. Lactoferrin and lysozyme deficiency in airway secretions: association with the development of bronchopulmonary dysplasia. J Pediatr 1992;121:262-70.

24. Bonten MJ, Bergmans DC, Ambergen AW, et al. Risk factors for pneumonia, and colonization of respiratory tract and stomach in mechanically ventilated ICU patients. Am J Respir Crit Care Med 1996;154:1339-46.

25. de Latorre FJ, Pont T, Ferrer A, et al. Pattern of tracheal colonization during mechanical ventilation. Am J Respir Crit Care Med 1995;152:1028-33.

26. Drakulovic MB, Bauer TT, Torres A, et al. Initial bacterial colonization in patients admitted to a respiratory intensive care unit: bacteriological pattern and risk factors. Respiration 2001;68:58-66.

27. Sirvent JM, Torres A, Vidaur L, et al. Tracheal colonisation within $24 \mathrm{~h}$ of intubation in patients with head trauma: risk factor for developing early-onset ventilator-associated pneumonia. Intensive Care Med 2000;26:1369-72.

28. Niederman MS, Mantovani R, Schoch P, et al.
Patterns and routes of tracheobronchial colonization in mechanically ventilated patients. The role of nutritional status in colonization of the lower airway by Pseudomonas species. Chest 1989;95:155-61.

29. Schwartz SN, Dowling JN, Benkovic C, et al. Sources of gram-negative bacilli colonizing the tracheae of intubated patients. J Infect Dis 1978;138:227-31.

30. Torres A, el-Ebiary M, González J, et al. Gastric and pharyngeal flora in nosocomial pneumonia acquired during mechanical ventilation. Am Rev Respir Dis 1993;148:352-7.

31. Adair CG, Gorman SP, Feron BM, et al. Implications of endotracheal tube biofilm for ventilator-associated pneumonia. Intensive Care Med 1999;25:1072-6.

32. Rello J, Sa-Borges M, Correa H, et al. Variations in etiology of ventilator-associated pneumonia across four treatment sites: implications for antimicrobial prescribing practices. Am J Respir Crit Care Med 1999;160:608-13.

33. Spencer RC. Predominant pathogens found in the European Prevalence of Infection in Intensive Care Study. Eur J Clin Microbiol Infect Dis 1996;15:281-5.

34. Kunac A, Sifri ZC, Mohr AM, et al. Bacteremia and ventilator-associated pneumonia: a marker for contemporaneous extra-pulmonic infection. Surg Infect (Larchmt) 2014;15:77-83.

35. Lampati L, Maggioni E, Langer M, et al. Can routine surveillance samples from tracheal aspirate predict bacterial flora in cases of ventilator-associated pneumonia? Minerva Anestesiol 2009;75:555-62.

36. Nseir S, Di Pompeo C, Pronnier P, et al. Nosocomial tracheobronchitis in mechanically ventilated patients: incidence, aetiology and outcome. Eur Respir J 2002;20:1483-9.

37. George DL, Falk PS, Wunderink RG, et al. Epidemiology of ventilator-acquired pneumonia based on protected bronchoscopic sampling. Am J Respir Crit Care Med 1998;158:1839-47.

38. Michel F, Franceschini B, Berger P, et al. Early antibiotic treatment for BAL-confirmed ventilator-associated pneumonia: a role for routine endotracheal aspirate cultures. Chest 2005;127:589-97.

39. Jung B, Sebbane M, Chanques G, et al. Previous endotracheal aspirate allows guiding the initial treatment of ventilator-associated pneumonia. Intensive Care Med 2009;35:101-7.

40. Luna CM, Sarquis S, Niederman MS, et al. Is a strategy based on routine endotracheal cultures the best way to prescribe antibiotics in ventilator-associated pneumonia? 
Chest 2013;144:63-71.

41. Brusselaers N, Logie D, Vogelaers D, et al. Burns, inhalation injury and ventilator-associated pneumonia: value of routine surveillance cultures. Burns 2012;38:364-70.

42. Brusselaers N, Labeau S, Vogelaers D, et al. Value of lower respiratory tract surveillance cultures to predict bacterial pathogens in ventilator-associated pneumonia: systematic review and diagnostic test accuracy meta-analysis. Intensive Care Med 2013;39:365-75.

43. Chan JD, Dellit TH, Choudhuri JA, et al. Active surveillance cultures of methicillin-resistant Staphylococcus aureus as a tool to predict methicillinresistant S. aureus ventilator-associated pneumonia. Crit Care Med 2012;40:1437-42.

44. Kollef MH. Inadequate antimicrobial treatment: an important determinant of outcome for hospitalized patients. Clin Infect Dis 2000;31:S131-8.

45. Leroy O, Meybeck A, d'Escrivan T, et al. Impact of

Cite this article as: Dray S, Coiffard B, Persico N, Papazian L, Hraiech S. Are tracheal surveillance cultures useful in the intensive care unit? Ann Transl Med 2018;6(21):421. doi: 10.21037/atm.2018.08.39 adequacy of initial antimicrobial therapy on the prognosis of patients with ventilator-associated pneumonia. Intensive Care Med 2003;29:2170-3.

46. Welch HK, Nagel JL, Patel TS, et al. Effect of an antimicrobial stewardship intervention on outcomes for patients with Clostridium difficile infection. Am J Infect Control 2016;44:1539-43.

47. Garnacho-Montero J, Gutiérrez-Pizarraya A, EscorescaOrtega A, et al. De-escalation of empirical therapy is associated with lower mortality in patients with severe sepsis and septic shock. Intensive Care Med 2014;40:32-40.

48. Kollef MH, Morrow LE, Niederman MS, et al. Clinical characteristics and treatment patterns among patients with ventilator-associated pneumonia. Chest 2006;129:1210-8.

49. Giantsou E, Liratzopoulos N, Efraimidou E, et al. Deescalation therapy rates are significantly higher by bronchoalveolar lavage than by tracheal aspirate. Intensive Care Med 2007;33:1533-40. 